\title{
Providing gender-affirming hormone therapy through primary care: service users' and health professionals' experiences of a pilot clinic
}

\author{
Alex Ker BA(Hons); ${ }^{1,5}$ Gloria Fraser BA, BSc(Hons); ${ }^{2}$ Antonia Lyons BA(Hons), PhD; ${ }^{3}$ Cathy Stephenson MBChB, \\ MRCGP, Dip Child Health, Dip Obst and Gynae, FRNZCGP; ${ }^{4}$ Theresa Fleming BA, Dip SW, MHSci, PhD ${ }^{3}$ \\ ${ }^{1}$ School of Social and Cultural Studies, Victoria University of Wellington, Wellington, New Zealand \\ ${ }^{2}$ School of Psychology, Victoria University of Wellington, Wellington, New Zealand \\ ${ }^{3}$ School of Health, Victoria University of Wellington, Wellington, New Zealand \\ ${ }^{4}$ Mauri Ora, Student Health and Counselling Service, Victoria University of Wellington, Wellington, New Zealand \\ ${ }^{5}$ Corresponding author. Email: alex.ker@vuw.ac.nz
}

J PRIM HEALTH CARE 2020;12(1):72-78. doi:10.1071/HC19040 Received 22 April 2019 Accepted 8 February 2020 Published 10 March 2020

\begin{abstract}
INTRODUCTION: Primary health care providers are playing an increasingly important role in providing gender-affirming health care for gender diverse people. This article explores the experiences of a primary care-based pilot clinic providing gender-affirming hormone therapy in Wellington, New Zealand.
\end{abstract}

AIM: To evaluate service users' and health professionals' experiences of a pilot clinic at Mauri Ora (Victoria University of Wellington's Student Health and Counselling Service) that provided genderaffirming hormones through primary care.

METHODS: In-depth interviews were conducted with four (out of six) service users and four health professionals about their perspectives on the clinic. Interviews were transcribed verbatim and analysed using thematic analysis.

RESULTS: Three themes were identified in service users' interviews, who discussed receiving affirming care due to the clinic's accessibility, relationship-centred care and timeliness. Three themes were identified in the health professionals' interviews, who described how the clinic involves partnership, affirms users' gender and agency, and is adaptable to other primary care settings. Both service users and health professionals discussed concerns about the lack of adequate funding for primary care services and the tensions between addressing mental health needs and accessing timely care.

DISCUSSION: The experiences of service users and health professionals confirm the value of providing gender-affirming hormone therapy in primary care. Models based in primary care are likely to increase accessibility, depathologise gender diversity and reduce wait times.

KEYWORDS: Gender-affirming health care; primary health care; transgender; LGBTQ persons; gender minorities

\section{Introduction}

In this paper, we use 'gender diverse' to refer to people whose gender differs from their assigned sex at birth. This term encompasses a range of gender identities and expressions, including transgender or trans, non-binary, takatāpui, whakawahine, tangata ira tāne and tāhine. 
An estimated $1.2 \%$ of young people in New Zealand (NZ) identify as gender diverse (their gender differs from their sex assigned at birth) and $2.5 \%$ report questioning their gender. ${ }^{1}$ Gender diverse people experience high rates of mental health difficulties because of social discrimination faced for challenging traditional binary gender norms. ${ }^{2,3}$ Many experience gender dysphoria, the distress caused by incongruence between a person's gender and their body. ${ }^{4}$ Hormone therapy can effectively reduce gender dysphoria and improve individuals' quality of life. ${ }^{5-8}$

There is a growing, yet unmet need for genderaffirming hormone therapy in NZ. Most recent findings indicate that $19 \%$ of gender diverse people in NZ want to access gender-affirming hormones but cannot because of high costs, fear of mistreatment or not knowing where to seek care. ${ }^{9}$ Referrals to the Wellington Endocrine Service for gender-affirming hormone therapy have been increasing since $2010,{ }^{10}$ but lack of allocated funding ${ }^{11}$ and shortage of qualified health professionals has led to long waiting times. A recent survey found that the average wait time between requesting and receiving hormone therapy in NZ was 46.9 weeks (unpubl. data). Waiting for genderaffirming care can have negative effects on people's mental health. ${ }^{12,13}$

As of 2018, pathways to accessing gender-affirming hormone therapy across District Health Boards (DHBs) in NZ were not transparent. Five of the 20 DHBs did not provide full funding for genderaffirming hormone therapy, including for readiness assessments. These assessments, typically conducted by a mental health professional (e.g. counsellor, psychologist) with credentials outlined in the World Professional Association for Transgender Health Standards of Care, version $7,{ }^{14}$ ensure that service users are fully informed of the personal and social implications of hormone therapy. Hormone therapy can be accessed privately, but at significant financial cost; in Wellington, a readiness assessment conducted by a private psychologist can cost up to $\$ 700-800$.

In January 2018, a clinic to provide genderaffirming hormone therapy was piloted at Mauri Ora, the Student Health and Counselling Service of Victoria University of Wellington. The clinic

\section{WHAT GAP THIS FILLS}

What is already known: The demand for gender-affirming hormone therapy is growing in New Zealand, but there is unmet need in many of the country's regions. International literature and guidelines for gender-affirming health care indicate that gender-affirming hormone therapy is effectively provided in primary care settings that are familiar and accessible to service users. However, little qualitative research has explored service users' and health professionals' experiences of primary care-based services.

What this study adds: This qualitative study centres on New Zealand health service users' experiences of gender-affirming health care in primary care settings. Findings are expected to provide clinicians with insight and knowledge about providing gender-affirming hormone therapy in primary care.

model, which is based on international guidelines, ${ }^{15,16}$ was developed by clinic staff, the regional Endocrine Service, community advisers and Capital and Coast DHB's Sex and Gender Diverse Working Group (a partnership between members experienced in planning and delivering youth health services and community members who provide health and support services within the genderdiverse community). A separate working group with representation from gender diverse students was also consulted around the clinic's development.

The six service users attended an average of five 1-h sessions with a counsellor, and two to four sessions with a GP to ensure there were no psychosocial or physical health issues that would be a barrier to starting hormone therapy. Where necessary, existing mental health issues were addressed during sessions with the counsellor. Gender-affirming hormones were subsequently prescribed at an appointment with the GP and endocrinologist, during which service users gave their informed consent. A clinic nurse, counsellor or student support person also sometimes attended this appointment. The average length of the clinic process from the first appointment to the point of receiving hormone therapy was 4 months. Service users were offered up to three follow-up GP appointments, and could choose to access further counselling support. The clinic ensured that service users would be transferred to other primary care services when no longer enrolled at Mauri Ora. 


\section{Methods}

Ethics approval was granted by the Victoria University of Wellington Human Ethics Committee. An independent Mauri Ora staff member invited the six service users to participate, while health professionals were invited by a member of the research team.

\section{Participants}

Four service users agreed to participate (two undergraduate students and two taking a break from study), aged $18-26$ years (mean age 20 years). Three identified their gender as female, and one identified as nonbinary and neutrois (not identifying as male or female). Three of the four health-care professionals who participated were members of the clinic's multidisciplinary team and one was a community member, counsellor and independent adviser to the clinic.

\section{Study procedures}

Seven individual interviews lasting 39-80 min (mean $=52 \mathrm{~min}$ ) were conducted on the university campus; one interview was conducted by telephone. All interviews were conducted and transcribed verbatim by the first author, a Sociology Honours student who identifies as gender diverse. Participants were each given a NZ\$30 voucher to thank them for their involvement in the research.

\section{Analysis}

In the first phase of analysis, the first author manually coded all transcripts into topic areas and category groups. Another researcher coded a subset of transcripts. Both sets of coding were checked for consistency, discrepancies identified and discussed until agreement was reached. In the second phase of analysis, all team members discussed ways the codes were similar conceptually, and from there, they were grouped into tentative themes. In the third stage of analysis, further analytic work was undertaken to identify key semantic themes ${ }^{17}$ and subthemes that addressed the research questions and captured the participants' accounts.

\section{Results}

Three themes were identified across each participant group, and one was identified across both groups. Each theme is described below.

\section{Service users' experiences}

Accessibility

Service users described how the accessibility of the clinic alleviated some stress in seeking care and enabled them to focus on making informed decisions. Mauri Ora was a familiar setting to all participants, which made it easier to make initial contact with the service:

'That was useful, you know, having a place I was familiar with and someone I was familiar with there, and also just being able to come up to campus and do it. It was much easier I think than - hospitals are scary!'

Other participants described the whole clinic process as 'smooth,' 'easy', 'comprehensive' and flexible:

'There was definitely a sense of flexibility ... [the staff] made it clear from early on that they would make it work which was very reassuring, and also giving me the time I needed in the process.'

The ease of accessing the clinic enabled service users to focus on making informed decisions about starting gender-affirming hormone therapy. As one participant explained, the easiest part of the clinic was that 'all the student has to do is focus on deciding whether [hormone therapy] is the right decision for them ... they don't have to worry about the logistics'. These views suggest that locating gender-affirming care 'close to home' may ease additional stress associated with seeking transition-related care.

\section{Relationship-centred care}

All service users described how developing positive therapeutic relationships with staff allowed them to talk openly about their health needs. One participant shared that:

'I had a good relationship with [the counsellor], and I always enjoyed talking to her and came away feeling better about whatever problems I had at the time.'

'Being able to explore those feelings in a safe and supportive environment and know that you can go on hormones if you want through this process would take a lot of stress off people.' 
In addition to having someone to talk to, participants also discussed the value of working with clinic staff who were affirming of gender diversity. As a result, some service users shared they did not feel pressure to 'prove' their gender identity:

'It was refreshing not to have to go through a gatekeeping process of saying, 'are you masculine enough or are you feminine enough?'. It was simply a matter of, 'you want hormones - okay, let's figure out the best way to give them to you."

For most participants, the perceived focus of the clinic was to provide service users with information and support, rather than to evaluate their gender. Enabling service users to make informed choices through person-centred care may increase their autonomy over their gender and health needs.

\section{Timeliness of care}

All service users described wait times for appointments in the pilot clinic favourably compared with their perceptions of wait times in secondary care. Most participants spoke about the urgency of accessing hormones for people who experience gender dysphoria, which suggests the need for timely care. As one service user explained:

'I think paradoxically even being on the waiting list [in secondary care] can make it worse because you're that much closer to your goal but there's a kind of arbitrary bureaucracy standing in the way between getting you the medication that you need to continue living.'

Another service user described valuing the clinic at Mauri Ora because people 'can get all these appointments within a short amount of time for free'. Another participant described the timeliness, ease and nature of the service as key factors they considered:

'The two things that made me want to do it were that it would be happening quicker than I could get access to hormone replacement therapy through the hospital system, and that it seemed like it would be an easier and friendlier process.'

Two participants described not feeling rushed into making decisions about hormone therapy after their first appointment. Two other participants (who required less support from staff in their decision-making) commented that fewer counselling sessions could save time and resources. While one participant shared that the number of sessions was 'probably more than would strictly be necessary to make sure people are comfortable with the process', two participants described the value of multiple counselling sessions. These differing support needs highlights the importance of individually tailoring care.

\section{Health professionals' experiences}

\section{Taking a 'partnership approach'}

Health professionals described the importance of partnership in the provision of gender-affirming hormone therapy, notably the multidisciplinary approach to care. One health professional viewed primary care as a more appropriate setting than secondary care to provide hormone therapy:

'[The multi-disciplinary approach is] certainly something that can be done far better in primary care than in secondary care because GPs are just so much better set up in terms of the environment and the support staff.'

Health professionals also discussed the importance of partnering with gender diverse communities to ensure that the clinic affirmed service users' gender and autonomy:

'The working groups here ... have been really key in terms of addressing that whole of service approach to this ... so that's been a really amazing partnership in terms of I guess opening our eyes to what we thought we were doing quite well and we probably weren't.'

Getting feedback from gender diverse students was essential for making the clinic environment accessible and affirming. Participants noted that gender diverse community organisations across NZ are doing vital work to support mental health and suicide prevention among gender diverse populations and can provide valuable input to the development of services.

\section{Affirming users' gender and agency}

Participants agreed that service users' gender can be affirmed better in primary than in secondary care because these services can be more welcoming and non-pathologising. One health professional 
emphasised that hospital settings do not tend to be affirming spaces for gender diverse service users:

'There's nothing in the [hospital] waiting room that supports [gender diversity] or says 'we're inclusive' or 'this is what we do'.'

The three clinicians described their role as supporting service users to make informed decisions about their own health, as they would for other health issues:

'It's my job to be informed and supporting [service users] to navigate all [aspects of transitioning], should they wish to do that. That you get given a range of options and choose one that suits you, just as you would if you were going to get breast screening or some other kind of healthcare.'

Participants noted that affirming gender was important because gender diverse people may enter services anticipating they may be denied care:

'[service users may be] a little bit worried that we're acting as gatekeeping service, and that we're going to come along and say 'no you can't do this' and all that sort of stuff, which isn't the case at all.'

Some professionals acknowledged that negative health-care experiences are still common among gender diverse people, and that perceptions of gatekeeping still exist. One participant acknowledged that the requirement for readiness assessments could be perceived as gatekeeping, but explained that clinic staff tried to minimise this power dynamic by affirming service users' agency throughout the process.

\section{Adaptability}

All professionals noted that the Mauri Ora approach to providing gender-affirming hormone therapy was adaptable to primary care settings, which is consistent with overseas models. Three professionals reported that the clinic was easy to implement at Mauri Ora because their current practice did not need to change much. One described the clinic as 'a really simple thing to get going' and was confident that this model would work 'anywhere in primary care, as long as the practice was safe and the provider was safe'. Most participants commented that this clinic model could be implemented in student health services, community health-care providers, and other places with multidisciplinary teams. As one participant noted, the clinic:

'... seemed to be something that other places had done quite well when there were multidisciplinary teams already ... that's really why we tried very hard to make it really simple and make it reproducible elsewhere and scalable.'

One participant mentioned that in adapting this model to other settings, services could consider how to best meet the needs of specific populations, particularly Māori, Pasifika and rural communities.

\section{Shared theme: Current concerns}

Service users and health professionals expressed concern about pressures on clinic staff due to a shortage of skilled practitioners and increasing numbers of people seeking hormone therapy. Although the clinic had capacity initially, it was expected that numbers of service users would increase. Both groups also discussed the tensions between addressing mental health difficulties and the timeliness of hormone therapy. While two service users commented that they valued the time taken to address their mental health difficulties, two others reported being cautious when talking about mental health with clinicians in case they were denied access to hormone therapy. Further, one service user described a tension between needing to access hormone therapy quickly while also addressing their mental health difficulties. This tension was also acknowledged by one health professional, who perceived that the clinic's structure '... dictate[d] the pace of the work with the client. That's a challenge - client pace versus clinic pace'. While the clinic reduced overall wait times, these tensions around time and mental health highlight some of the complexities around providing affirming care to a diverse population who tend to experience higher rates of mental health challenges.

\section{Discussion}

There is a dearth of research on gender diverse people's experiences of accessing gender-affirming health care in NZ settings. However, recent findings on service user satisfaction overseas indicate that providing gender-affirming care through 
multidisciplinary teams can reduce barriers and provide individually tailored support. ${ }^{18,19}$ Similarly, our study showed that a primary care-based model reduced service users' wait times for genderaffirming hormone therapy. Participants noted how the clinic aimed to remove unnecessary barriers by providing care in an environment 'close to home', consistent with the New Zealand Guidelines for Gender Affirming Healthcare. ${ }^{4}$

This study's findings offer insight into how service users understand gatekeeping and informed consent. Gatekeeping has been identified as a key issue when accessing gender-affirming care. ${ }^{20,21}$ The clinic was developed in line with other Capital and Coast DHB services, which currently follow recommendations of the World Professional Association for Transgender Health to obtain a readiness letter from a qualified mental health professional. ${ }^{14}$ Although most participants in this study did not perceive the clinic staff as gatekeepers, one service user noted that the requirement to see a counsellor may still be perceived as a barrier. Some services overseas are attempting to remove such barriers by assessing mental health needs in GP appointments, then offering extra support as required. ${ }^{16}$ Currently, informed consent for gender-affirming care is not standardised across NZ. The revision of the World Professional Association for Transgender Health's Standards of Care, due in 2020, may offer clearer guidelines for establishing informed consent models and reducing gatekeeping. The sample in this exploratory study was relatively homogenous. The four service users were all university students and had access to free or reduced-cost health care. Older adults, adolescents and children may have different health needs, which need to be considered when implementing similar models. The health professionals were selected for their varied involvement with the clinic, but it would be beneficial to obtain a wider set of professional views. Despite these limitations, this qualitative evaluation does offer important insights into the benefits of providing gender-affirming primarybased care in NZ.

In future studies, researchers could compare service users' experiences of primary and secondary care models, as well as their experiences of wait times. It will also be important to consider how a similar model might be implemented in rural health-care settings with potentially fewer resources. If future research found further support for providing hormone therapy in primary care, providers working in established multidisciplinary team models such as youth one-stop shops, tertiary education health services and community health-care providers, could adopt a similar model.

\section{Author information}

Alex Ker, Honours student, School of Social and Cultural Studies, Victoria University of Wellington, Wellington; Gloria Fraser, PhD student, School of Psychology, Victoria University of Wellington, Wellington; Antonia Lyons, Professor, School of Health, Victoria University of Wellington, Wellington; Terry Fleming, Senior Lecturer, School of Health, Victoria University of Wellington, Wellington; Cathy Stephenson, General Practitioner, Mauri Ora, Student Health and Counselling Service, Victoria University of Wellington, Wellington.

\section{Competing interests}

The authors declare no competing interests.

\section{Acknowledgements}

We would like to thank participants for sharing their experiences, the Sex and Gender Diverse Working Group, students at Victoria University of Wellington community groups and NGOs who support gender diverse communities in Aotearoa; Richard Carroll, Jemima Bullock, Anny da Silva Freitas, Tommy Hamilton, staff at Mauri Ora and all health-care professionals who were involved in the pilot clinic. We would also like to acknowledge and thank the Faculty of Health for funding a research position for this study.

\section{References}

1. Clark TC, Lucassen MFG, Bullen $P$, et al. The health and well-being of transgender high school students: results from the New Zealand Adolescent Health Survey (Youth'12). J Adolesc Health. 2014;55:93-9. doi:10.1016/j.jadohealth. 2013.11.008

2. Bockting WO, Miner MH, Swinburne Romine RE, et al. Stigma mental health, and resilience in an online sample of the US transgender population. Am J Public Health. 2013;103:94351. doi:10.2105/AJPH.2013.301241

3. White Hughto JM, Reisner SL, Pachankis JE. Transgender stigma and health: a critical review of stigma determinants, mechanisms, and interventions. Soc Sci Med. 2015;147:22231. doi:10.1016/j.socscimed.2015.11.010 
4. Oliphant J, Veale J, Macdonald J, et al. Guidelines for gender affirming healthcare for gender diverse and transgender children, young people and adults in Aotearoa, New Zealand Transgender Health Research Lab, University of Waikato: New Zealand; 2018.

5. White Hughto JM, Reisner SL. A systematic review of the effects of hormone therapy on psychological functioning and quality of life in transgender individuals. Transgend Health. 2016;1:21-31. doi:10.1089/trgh.2015.0008

6. Colton Meier S, Fitzgerald K, Pardo S, Babcock J. The effects of hormonal gender affirmation treatment on mental health in female-to-male transsexuals. J Gay Lesbian Ment Health. 2011;15:281-99. doi:10.1080/19359705.2011.581195

7. Costa R, Colizzi M. The effect of cross-sex hormonal treatment on gender dysphoria individuals' mental health: a systematic review. Neuropsychiatr Dis Treat. 2016;12:1953-66. doi:10. 2147/NDT.S95310

8. Murad MH, Elamin MB, Garcia MZ, et al. Hormonal therapy and sex reassignment: a systematic review and meta-analysis of quality of life and psychosocial outcomes. Clin Endocrinol (Oxf). 2010;72:214-31. doi:10.1111/j.1365-2265.2009.03625.x

9. Veale J, Byrne J, Tan K, et al. Counting ourselves: the health and wellbeing of trans and non-binary people in Aotearoa New Zealand. Hamilton, NZ: Transgender Health Research Lab, University of Waikato; 2019 [cited 2019 September 24]. Available from: https://countingourselves.nz/

10. Delahunt JW, Denison HJ, Sim DA, et al. Increasing rates of people identifying as transgender presenting to Endocrine Services in the Wellington region. NZMed J. 2018;131:33-42.

11. Professional Association for Transgender Health Aotearoa [PATHA]. Is the provision of gender affirming health care equitable across the District Health Boards in Aotearoa, New Zealand? PATHA; 2019 [cited 2019 September 25]. Available from: https://patha.nz/news/is-the-provision-of-genderaffirming-health-care-equitable-across-the-district-healthboards-in-aotearoa-newzealand
12. Gridley SJ, Crouch JM, Evans Y, et al. Youth and caregiver perspectives on barriers to gender-affirming health care for transgender youth. J Adolesc Health. 2016;59:254-61. doi:10.1016/j.jadohealth.2016.03.017

13. Cohen-Kettenis PT, Delemarre-van de Waal HA, Gooren LJ. The treatment of adolescent transsexuals: changing insights. J Sex Med. 2008;5:1892-7. doi:10.1111/j.1743-6109.2008. 00870.x

14. WPATH. Standards of Care for the Health of Transsexual, Transgender, and Gender Nonconforming People. Version 7. World Professional Association for Transgender Health; 2012; pp. 1-112.

15. Equinox. Protocols for the initiation of hormone therapy for trans and gender diverse patients; 2017 [cited 2019 September 25]. Available from: https://equinox.org.au/ resources/

16. Schulz S. The informed consent model of transgender care: an alternative to the diagnosis of gender dysphoria. J Humanist Psychol. 2018;58:72-92. doi:10.1177/0022167817745217

17. Braun V, Clarke V. Using thematic analysis in psychology. Qual Res Psychol. 2006;3:77-101. doi:10.1191/ 1478088706qp063oa

18. Tollit MA, Feldman D, McKie G, Telfer MM. Patient and parent experiences of care at a pediatric gender service. Transgend Health. 2018;3:251-6. doi:10.1089/trgh.2018.0016

19. Inwards-Breland DJ, DiVall S, Salehi P, et al. Youth and parent experiences in a multidisciplinary gender clinic. Transgend Health. 2019;4:100-6. doi:10.1089/trgh.2018.0046

20. Cavanaugh T, Hopwood R, Lambert C. Informed consent in the medical care of transgender and gender-nonconforming patients. AMA J Ethics. 2016;18(11):1147-55.

21. Wi-Hongi A, Grieg A, Hazenberg E. Towards a model of informed consent: trans healthcare in Aotearoa New Zealand. In: Representing Trans. Meyerhoff M and Hazenberg E, ed. Wellington: Victoria University Press; 2017. pp. 153-170. 